\title{
Digging in the Dark Triad: A Replication of "Machiavellianism and Dating: Deception and Intimacy"
}

\author{
Guillaume Durand ${ }^{\mathrm{a}, \otimes}$ \\ ${ }^{a}$ Department of Psychiatry and Neuropsychology, Maastricht University
}

\begin{abstract}
The present study is a replication and an extension of Dussault, Hojjat, and Boone (2013). Machiavellianism and dating: Deception and intimacy. Social Behavior and Personality: an international journal, 41(2), 283-294. The results support the positive association between Machiavellianism and the use of deceptive dating strategies, but fail to detect a correlation between Machiavellianism and perceived intimacy. The extension to the Dark Triad reveals that psychopathy is a stronger predictor than Machiavellianism to assess deceptive dating strategies, and supports a relationship between higher degrees of narcissism and history of intimate behaviors.
\end{abstract}

Keywords a Replication, Machiavellianism, Dating, Dark Triad, Deception, Intimacy.

Acting Editor $\square \mathrm{De}$ nis Cousineau (Université d’Ottawa)

Reviewers

- One anonymous reviewer

- The Editor was unable to get an evaluation from the authors of the replicated study .

GD: 0000-0002-5418-4429

10.20982/tqmp.12.3.r006

\section{Introduction}

The 'Dark Triad of Personalities' refers to Machiavellianism, narcissism, and psychopathy; a set of three similar yet different personality disorders, categorized by socially malevolent tendencies, egoism, emotional coldness, and aggressive personality (Paulhus \& Williams, 2002). While sharing similarities, each of these constructs possesses its unique characteristics. Machiavellianism refers to social manipulation, a lack of concern for morality, and a desire to exploit others (Christie \& Geis, 1970). Narcissism refers to elevated display of arrogance, feelings of entitlement, and grandiosity (Maxwell, Donnellan, Hopwood, \& Ackerman, 2011). Psychopathy refers to a combination of emotional dysfunctions (e.g., reduced guilt, empathy and attachment) and antisocial behavior (e.g., impulsivity and aggressive tendencies)(Berg et al., 2013; Blair \& Mitchell, 2009). While several theories add an adaptive component to narcissism and psychopathy (e.g., Lilienfeld \& Widows, 2005; Pincus \& Lukowitsky, 2010), the Dark Triad refers to the pathological component of the aforementioned disorders (Paulhus \& Williams, 2002).

Several studies investigated the influence of Machiavellianism in interpersonal relationships. Findings support a relationship between higher levels of Machiavellianism and the use of deceptive mating strategies (e.g., blatant lying, self-praising and avoiding confrontation), as well as with the intentions to engage in infidelity (Brewer \& Abell, 2015). These results go in line with yet another study, reporting a negative relationship between Machiavellianism and the importance of intimacy and loyalty in their ideal relationships (Ináncsi, Láng, \& Bereczkei, 2016). Furthermore, a subsequent research concluded that individuals high on Machiavellianism will seek symbiotic closeness with their partners in order to extend their control over time and manipulate them with better ease (Ináncsi, Láng, \& Bereczkei, 2015). These findings support the manipulative tendencies of Machiavellian individuals in their intimate relationships.

In order to further investigate the role of Machiavellianism in interpersonal relationships, Dussault, Hojjat, and Boone (2013) recruited 123 undergraduate students to examine the relationship between Machiavellianism, deceptive mating strategies, intimacy, and commitment. The authors concluded that higher scores on Machiavellianism were associated with an increase of likelihood to use deceptive tactics to attract potential mates, as well as with lower levels of relationship intimacy, and proposed to replicate the findings using the Dark Triad instead of Machiavellianism alone. The purpose of this study is to 
replicate the previous findings confirming a relationship between higher Machiavellianism, higher deception, and lower intimacy, as well as to determine if this relationship applies to the whole Dark Triad.

\section{Method}

\section{Participants}

A total of 106 participants from the community (50 males and 56 females) were recruited for this online study. Participants were primarily located in Europe (57\%), America $(28 \%)$, Asia (8\%), Oceania (6\%) or Africa (1\%). The most common education levels completed by the participants (or the current level for students) were undergraduate degree (54\%), Master's degree (26\%), Doctoral degree (7\%), or other (13\%). Participants ranged in age from 18 to $74(\mathrm{M}=$ $26.58, \mathrm{SD}=9.48$ ). All participants provided informed consent prior to filling out the questionnaires.

\section{Measures}

The procedure for this experiment was the same as the one used by Dussault et al. (2013), with the exception of the instrument used to assess Machiavellian traits. In the original study, the authors employed the Machiavellian IV Scale (Mach-IV), a 20-item scale assessing Machiavellianism (Christie \& Geis, 1970). In order to control for Machiavellianism, but also to expend to the full Dark Triad, I replaced the Mach-IV by the Short Dark Triad (SD3), a 27item self-report questionnaire assessing Machiavellianism, narcissism and psychopathy (Jones \& Paulhus, 2014). Each component is assessed by nine questions, ranging from 1 (Strongly Disagree) to 5 (Strongly Agree). The 26-item Intimate Relationship Questionnaire (IRQ; Sims-Knight, personal communication, March 27, 2007) which includes two components, namely perception of intimacy (PI), evaluating the participant's perceptions of intimacy in his relationships, and history of intimate behaviors (HIB), evaluating previous relationships in term of interests in pursing romantic or sexual relationships, commitment, sexual intimacy, and fidelity, remained unchanged from the original study. The 41-item Modified Relationship Strategies Questionnaire (Tooke \& Camire, 1991, DMS;), measuring the use of deceptive tactics in order to attract potential mates also remained unchanged. Descriptive data of the three questionnaires from the present study can be found in Table 1.

\section{Results}

In order to replicate previous findings regarding the relationship between DMS and Machiavellianism, gender, HIB, PI, as well as to determine the relationship of DMS with narcissism and psychopathy, a Zero-Order correlation analysis was performed (see Table 2). In the present study,
DMS showed a moderate positive correlation with Machiavellianism and psychopathy. Gender was also positively correlated with Machiavellianism and psychopathy, but not or weakly negatively correlated with PI and HIB. These results show that, while males tend to display higher levels of Machiavellianism and psychopathy, they will display lower levels of intimacy than females. Due to the conceptual relationship within the constructs of the Dark Triad, Machiavellianism, narcissism, and psychopathy were all positively correlated with one another. Furthermore, narcissism displayed a weak correlation with HIB, but not with PI. Lastly, as two measures of intimacy, HIB and PI displayed a positive correlation.

Three regression models were computed in order to determine the predictive value of each variable on deceptive mating strategies, history of intimate behaviors, and perceptions of intimacy (Table 3). The first model, with DMS as a dependent variable, was significant $(F(6,105)=6.068$, $p<.001, R^{2}=.27$ ). Closer examination of the model shows that gender and psychopathy (being a female and displaying higher levels of psychopathy) was related to DMS. Machiavellianism, while almost significant ( $p=.07$ ), did not load on the model. The second model, with PI as a dependent variable, was significant $(F(6,105)=6.794$, $p<.001, R^{2}=.29$ ). Only one variable was significant in this model, which was the history of intimate behaviors. The third model, with HIB as a dependent variable, was also significant $\left(F(6,105)=6.212, p<.001, R^{2}=.27\right)$. Once again, there was only one significant variable in this model, namely perceptions of intimacy.

\section{Discussion}

Overall, this study partially replicates the previous findings of (Dussault et al., 2013). The results confirm a relationship between Machiavellianism and DMS, Machiavellianism and gender, and PI and HIB. However, no support was found for the previously reported relationship between PI and Machiavellianism, or PI and DMS, indicating that intimacy was not correlated with Machiavellianism or deceptive mating strategies. Furthermore, by extending the study to the Dark Triad, I found a relationship between DMS and psychopathy, gender and psychopathy, and narcissism and HIB. The impact of psychopathy was further investigated, and results from the first regression model support the influence of psychopathy over Machiavellianism when predicting deceptive mating strategies. These findings demonstrate the overlapping nature of psychopathy and Machiavellianism. Conceptually, the key component of Machiavellianism is manipulation, which is an important component when using deceptive tactics (Dussault et al., 2013). Considering that levels of psychopathy are more predictive of deceptive strategies than Machiavellianism, it 
Table 1 a Means, standard deviations, and alpha coefficients $(\mathrm{N}=106)$

\begin{tabular}{lrrc}
\hline Scales & Mean & SD & Alpha \\
\hline Short Dark Triad & & & \\
$\quad$ Machiavellianism & 3.15 & 0.77 & .82 \\
$\quad$ Narcissism & 2.58 & 0.61 & .67 \\
$\quad$ Psychopathy & 2.17 & 0.65 & .74 \\
$\quad$ Intimate Relationship Questionnaire & & & \\
$\quad$ Perceptions of intimacy (PI) & 80.84 & 13.33 & .87 \\
$\quad$ History of intimate behaviors (HIB) & 24.01 & 3.76 & .50 \\
$\quad$ Modified Relationship Strategies Questionnaire & & & \\
$\quad$ Deceptive Mating Strategies (DMS) & 91.84 & 30.10 & .92 \\
\hline
\end{tabular}

Table 2 - Zero-Order correlations for Deceptive Mating Strategies, Gender, Machiavellianism, Narcissism, Psychopathy, History of Intimate Behaviors, and Perceptions of Intimacy

\begin{tabular}{|c|c|c|c|c|c|c|}
\hline Variable & 1 & 2 & 3 & 4 & 5 & 6 \\
\hline DMS & .02 & $.37 * * *$ & .10 & $.46 * * *$ & -.11 & .09 \\
\hline \multicolumn{7}{|l|}{ Predictor Variable } \\
\hline 1. Gender & - & $.25 * *$ & -.04 & $.36 * * *$ & $-.22 *$ & -.03 \\
\hline 2. Machiavellianism & & - & $.20 *$ & $.52 * * *$ & -.07 & .18 \\
\hline 3. Narcissism & & & - & $.26 * *$ & .14 & $.22 *$ \\
\hline 4. Psychopathy & & & & - & -.18 & .07 \\
\hline 5. PI & & & & & - & $.46 * * *$ \\
\hline 6. HIB & & & & & & - \\
\hline
\end{tabular}

Note. ${ }^{*}: p<.05,{ }^{* *}: p<.01,{ }^{* * *}: p<.001$.

is possible that Machiavellian tendencies are a subtype of psychopathy rather than a personality disorder on its own.

The relationship between Narcissism and HIB provides additional insight on the role of the Dark Triad in mating strategies. Individuals displaying higher levels of narcissism tend to be very interested in sexual or romantic relationship, be involved in long lasting relationship, valorize sexual contacts with their partner, and are less likely to be infidel. This positive correlation goes against the regular conception of narcissism being related to short-lasting relationships (Paulhus \& Williams, 2002). Nevertheless, the current study confirms the role of Machiavellianism on deceptive mating strategies, and provides preliminary results on the relationship between the other Dark Triad personality disorders and various dating-related behaviors, such as deception and intimacy.

\section{References}

Berg, J. M., Smith, S. F., Watts, A. L., Ammirati, R., Green, S. E., \& Lilienfeld, S. O. (2013). Misconceptions regarding psychopathic personality: implications for clinical practice and research. Neuropsychiatry, 3, 63-74. doi:10.2217/npy.12.69
Blair, R. J. R. \& Mitchell, D. G. V. (2009). Psychopathy, attention and emotion. Psychological Medicine, 39(4), 54355. doi:10.1017/S0033291708003991

Brewer, G. \& Abell, L. (2015). Machiavellianism and sexual behavior: motivations, deception and infidelity. Personality and Individual Differences, 74, 186-191. doi:10.1016/j.paid.2014.10.028

Christie, R. \& Geis, F. (1970). Studies in machiavellianism. London: Academic Press.

Dussault, M., Hojjat, M., \& Boone, R. T. (2013). Machiavellianism and dating: deception and intimacy. Social Behavior and Personality, 41(2), 283-294. doi:10.2224/ sbp.2013.41.2.283

Ináncsi, T., Láng, A., \& Bereczkei, T. (2015). Machiavellianism and adult attachment in general interpersonal relationships and close relationships. Europe's Journal of Psychology, 11(1), 139-154. doi:10.5964/ejop.v11i1. 801

Ináncsi, T., Láng, A., \& Bereczkei, T. (2016). A darker shade of love: machiavellianism and positive assortative mating based on romantic ideals. Europe's Journal of Psychology, 12(1), 137-152. doi:10.5964/ ejop.v12i1 . 1007 
Table 3 a Models summaries

\begin{tabular}{lrrrr}
\hline Scale & Std. Error & Std. $\beta$ & $t$ & Sig. \\
Model 1: Regression model predicting DMS & & & & \\
\hline Gender & 5.66 & -.20 & -2.07 & .04 \\
Mach & 4.03 & .19 & 1.83 & .07 \\
Narci & 4.58 & -.06 & -.65 & .52 \\
Psycho & 5.02 & .43 & 3.90 & .00 \\
PI & .23 & -.08 & -.79 & .43 \\
HIB & .81 & .07 & .68 & .50 \\
Model 2: Regression model predicting PI & & & & \\
\hline Gender & 2.49 & -.15 & -1.57 & .12 \\
Mach & 1.79 & -.03 & -.32 & .75 \\
Narci & 1.99 & .09 & .94 & .35 \\
Psycho & 2.33 & -.14 & -1.18 & .24 \\
DMS & .04 & -.08 & -.79 & .43 \\
HIB & .31 & .31 & 5.25 & .00 \\
Model 3: Regression model predicting HIB & & & & \\
\hline Gender & .72 & .04 & .46 & .65 \\
Mach & .51 & .15 & 1.44 & .15 \\
Narci & .57 & .11 & 1.23 & .22 \\
Psycho & .67 & .01 & .04 & .97 \\
DMS & .01 & .07 & .68 & .50 \\
PI & .03 & .47 & 5.25 & .00 \\
\hline
\end{tabular}

Note . Mach = Machiavellianism; Narci = Narcissism; Psycho = Psychopathy; PI = Perceptions of intimacy; HIB = History of intimate behaviors; DMS = Deceptive mating strategies; Std. Error = Standard error; Std. $\beta=$ Standardized beta; sig. = signification.

Jones, D. N. \& Paulhus, D. L. (2014). Introducing the short dark triad (sd3) a brief measure of dark personality traits. Assessment, 21(1), 28-41.

Lilienfeld, S. \& Widows, M. (2005). Psychopathic personality inventory-revised: professional manual. Lutz, FL: Psychological Assessment Resources.

Maxwell, K., Donnellan, M. B., Hopwood, C. J., \& Ackerman, R. A. (2011). The two faces of narcissus? an empirical comparison of the narcissistic personality inventory and the pathological narcissism inventory. Personality and Individual Differences, 50(5), 577-582. doi:10. 1016/j.paid.2010.11.031
Paulhus, D. L. \& Williams, K. M. (2002). The dark triad of personality: narcissism, machiavellianism, and psychopathy. Journal of Research in Personality, 36(6), 556-563. doi:10.1016/S0092-6566(02)00505-6

Pincus, A. L. \& Lukowitsky, M. R. (2010). Pathological narcissism and narcissistic personality disorder. Annual Review of Clinical Psychology, 6, 421-446. doi:10.1146/ annurev.clinpsy.121208.131215

Tooke, W. \& Camire, L. (1991). Patterns of deception in intersexual and intrasexual mating strategies. Ethology and Sociobiology, 12(5), 345-364. doi:10.1016/0162 3095(91)90030-T

\section{Citation}

Durand, G. (2016). Digging in the dark triad: a replication of "machiavellianism and dating: deception and intimacy". The Quantitative Methods for Psychology, 12(3), r6-r9. doi:10.20982/tqmp.12.3.r006

Copyright @ 2016, Durand. This is an open-access article distributed under the terms of the Creative Commons Attribution License (CC BY). The use, distribution or reproduction in other forums is permitted, provided the original author(s) or licensor are credited and that the original publication in this journal is cited, in accordance with accepted academic practice. No use, distribution or reproduction is permitted which does not comply with these terms.

Received: 10/09/2016 Accepted: 19/09/2016 